\title{
Study of Proton Helicity Structure in Polarized $p+p$ Collisions at PHENIX
}

\author{
Kensuke Okada ${ }^{1}$ for the PHENIX collaboration \\ 1- RIKEN-BNL Research Center \\ BNL Bldg.510A Upton NY,11973
}

\begin{abstract}
In this report, we describe a study of proton helicity structure in polarized $p+p$ collisions at PHENIX. Up until now, we have focused on $\pi^{0}$ measurements. The asymmetry measurement rejects the maximum gluon polarization scenario. With higher integrated luminosity, various probes will provide complementary results.
\end{abstract}

\section{Introduction}

Polarized lepton-nucleon deep inelastic scattering (DIS) experiments revealed that only $25 \%$ of proton spin is carried by quarks and anti-quarks. A principal goal of the spin program at RHIC [2] is to determine the gluon spin contribution to the proton spin, using longitudinally polarized proton collisions where the gluon in the polarized proton interacts in leading order process. The evidence of gluon polarization should appear in the double helicity production asymmetries $\left(A_{L L}\right)$ shown in Eq.1, where $P$ is polarization of proton beam, and $\sigma_{++(+-)}$is the production rate in beam polarization direction of $++(+-)$.

$$
A_{L L} \equiv \frac{1}{P^{2}} \cdot \frac{\sigma_{++}-\sigma_{+-}}{\sigma_{++}+\sigma_{+-}}
$$

\section{PHENIX experiment}

The PHENIX detector is composed of central arms $\left(|\eta|<0.35, \phi: 2^{*} 0.5 \pi\right)$ and muon arms $(1.2<|\eta|<2.4, \phi: 2 \pi)$. In 2006, a forward electromagnetic calorimeter was newly installed. The collision information is provided by the beam-beam counter (BBC) and the zero degree counter (ZDC). Those are also used as luminosity monitors. The detailed description of the PHENIX detector can be seen elsewhere [3]. The longitudinal spin program was started in 2003. In 2006, PHENIX recorded collision data at the energy of $\sqrt{s}=200 \mathrm{GeV}$ for the total integrated luminosity of $7.5 \mathrm{pb}^{-1}$ with the polarization of $\sim 60 \%$.

\section{Cross section measurements of single particles}

Before extracting the asymmetry (Eq.1), it is important to confirm the applicability of our theoretical baseline with the cross section measurement. We published several cross section measurements of single particle production[4]. The integrated luminosity was calculated from the BBC counts. The conversion factor is obtained via the van der Meer scan technique [5].

For example, neutral pion, direct photon, and single electron (a representative of charm/bottom hadrons) are interesting probes for the gluon measurement. Factorized perturbative QCD calculations describe our measurements well. 


\section{Spin asymmetry measurement at RHIC}

At RHIC, the key feature to reduce the systematic uncertainty is in our bunch structure of the proton beams. They consist of 120 bunches and the revolution time is only 1.2 micro seconds. Each bunch has different polarization direction. By using the rapidly changing combinations of polarization direction, we can reduce the systematics of detector instability in the asymmetry measurement. However the characteristics of each bunch crossing are not identical. One obvious factor is luminosity. To normalize the luminosity difference, the BBC is used again. Since the BBC detects the same hard scattering interaction, it is important to confirm if there is no asymmetry in the BBC measurement itself. For this purpose, we checked a relative difference to ZDC counts. Currently the statistics of ZDC counts determines the uncertainty of relative luminosity measurement. One way to check the uncertainty related to bunch characteristics is to confirm null asymmetry by assigning random polarization direction patterns.

\subsection{Asymmetry measurement in $\pi^{0}$ production}

The neutral pion $\left(\pi^{0}\right)$ is a suitable probe of gluons in the proton for PHENIX, for the following reasons.

- In the low $p_{T}$ region, the dominant process is gluon-quark scattering.

- It is the most common particle in the hadron final state.

- PHENIX has a finely granulated central electromagnetic calorimeter with a triggering feature.

Figure 1 shows our preliminary $\pi^{0}$ asymmetry result as a function of $p_{T}^{\pi^{0}}$. The points are from the data with integrated luminosity of $1.8 \mathrm{pb}^{-1}$ in Run5 (2005) and $7.5 \mathrm{pb}^{-1}$ in Run6 (2006). Only high $p_{T}$ points are included from Run6 fast track analysis. The theory curves in the figure are GRSV curves [6]. Figure 2 shows the $\chi^{2}$ values as a function of $\Delta \mathrm{G}$ (at $Q^{2}=1 \mathrm{GeV}^{2}$ ) in the theory. The maximum $\Delta \mathrm{G}$ scenario is rejected. It should be noted that there is no uncertainty for the model assumption included.

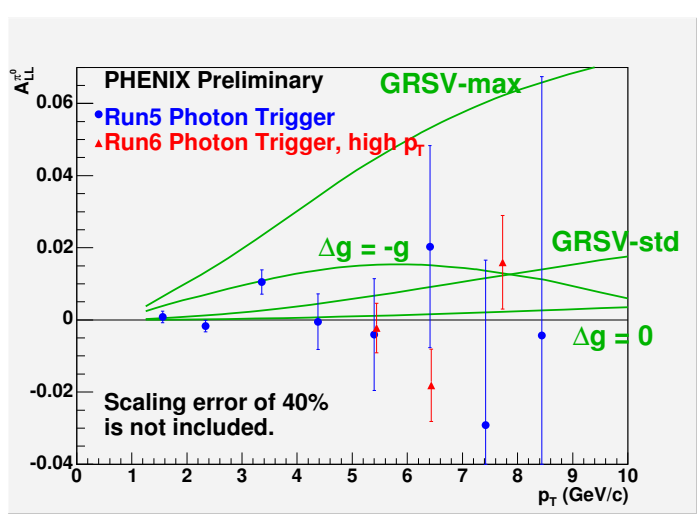

Figure 1: $A_{L L}^{\pi^{0}} \cdot$ Run6 data includes only $p_{T}$ higher than $5 \mathrm{GeV} / c$

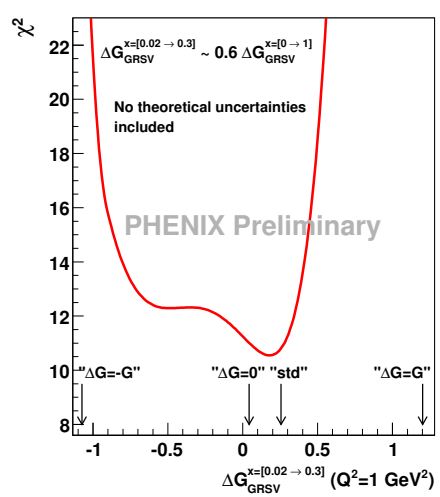

Figure 2: Data-Theory comparison $\left(\chi^{2}\right)$ plot. 
With a different collision energy, a different kinematic region can be investigated. For example, in $\sqrt{s}=62 \mathrm{GeV}$, the production cross section is about a hundred times larger than in $\sqrt{s}=200$ $\mathrm{GeV}$ in terms of $x_{T}\left(\equiv 2 p_{T} / \sqrt{s}\right)$, while the theory predicts $A_{L L}$ to be almost scaled. Although the luminosity is down by an order due to the larger emittance, it still wins at the higher $x_{T}$ region. Figure 3 shows the measurement from Run6 $\sqrt{s}=62 \mathrm{GeV}$ data $\left(\int L=\right.$ $\left.0.06 \mathrm{pb}^{-1}\right)$. The applicability of pQCD calculation is to be checked with cross section measurement.

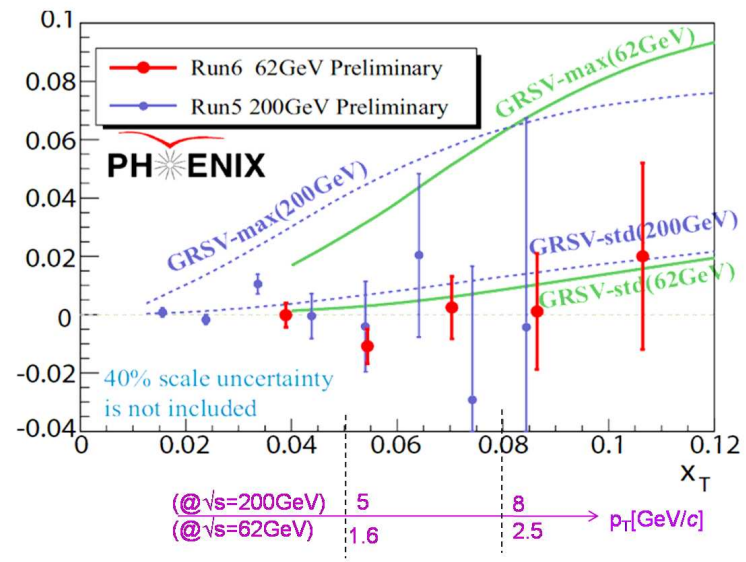

Figure 3: $\pi^{0} A_{L L}$ at $\sqrt{s}=62 \mathrm{GeV}$ as a function of $x_{T}\left(\equiv 2 p_{T} / \sqrt{s}\right)$.

\subsection{Asymmetry measurement in various channels}

As the integrated luminosity is increased, channels other than $\pi^{0}$ come into view.

\subsubsection{The central arm}

Figure 4 gives a rough idea of yields of several channels in the PHENIX central arm, and shows a statistical uncertainties of asymmetry measurement in two different conditions.

$\pi^{ \pm}$: In the $p_{T}$ range where gluon-quark scattering is dominant, the charge difference of this probe is thought to be sensitive to the sign of gluon polarization through the struck quark's favored/disfavored fragmentation function. Though the production probability is the same to $\pi^{0}$, the current PHENIX setup has a little

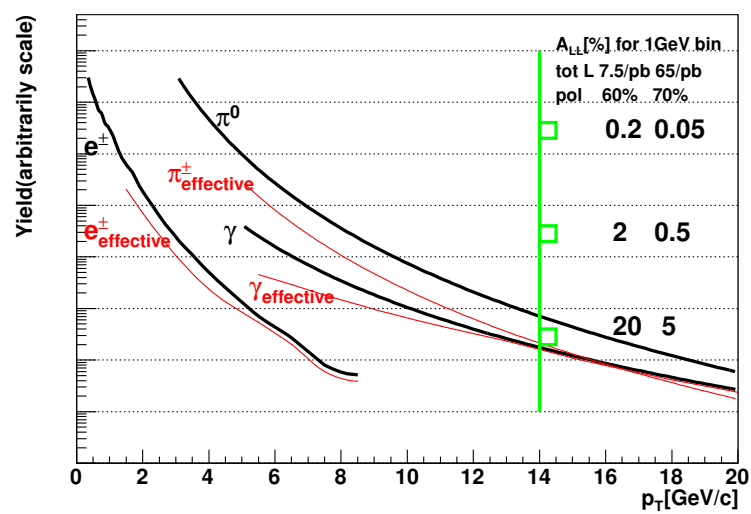
disadvantage in the trigger and the tracking system.

Direct $\gamma$ : In the theoretical point of view, this is a golden channel for gluons through the 
gluon Compton scattering process. However in addition to its small production probability, there are background photons from hadronic decay (mostly $\pi^{0}$ 's), which dilute the signal.

$e^{ \pm}$: The electron is a representative of charm or bottom particles. Since those heavy particles are produced through the gluon fusion process, it could be a good probe for gluon. The experimental disadvantage to $\pi^{0}$ is the limitation of data acquisition rate in the low $p_{T}$ region $\left(p_{T}<\sim 1.5 \mathrm{GeV} / c\right)$ and contamination of conversion electrons.

PHENIX has also asymmetry results of $\eta$ and "jet" production.

\subsubsection{The muon arm}

In the muon arm, the asymmetry of $J / \psi$ production with di-muon decay mode has been measured. It is starving for statistics. On the other hand, the detector is sensitive to hadron production with decay muons and punch-through hadrons.

\subsubsection{The forward electromagnetic calorimeter (MPC)}

It has a clear signal of $\pi^{0}$. It will provide information of $\pi^{0}$ production asymmetry in the large rapidity region.

\subsubsection{A different double spin asymmetry measurement}

One of ongoing analyses is to measure the spin dependence of dijet $k_{T}$ using two particle correlations $\left(\pi^{0}-h^{ \pm}\right)$. It has been proposed to have a sensitivity to the angular momentum component from an analogy of classical spinning disks [7]. It requires theoretical supports.

\section{Summary}

For the longitudinal spin program, the PHENIX experiment accumulated about $7.5 \mathrm{pb}^{-1}$ of data at $\sqrt{s}=200 \mathrm{GeV}$ with roughly $60 \%$ proton beam polarization and $0.1 \mathrm{pb}^{-1}$ of data at $\sqrt{s}=62 \mathrm{GeV}$ with $50 \%$ polarization. Up until now, we have performed spin asymmetry measurement focusing on $\pi^{0}$ production with the central electromagnetic calorimeters. Despite low integrated luminosity, it was shown that the $\sqrt{s}=62 \mathrm{GeV}$ data have comparable sensitivity to the gluon polarization for the high $x_{T}\left(\equiv 2 p_{T} / \sqrt{s}\right)$ region.

The longitudinal polarization program at $\sqrt{s}=200 \mathrm{GeV}$ will be accomplished with about $70 p b^{-1}$ and $70 \%$ polarization in 2008. With higher statistics, various probes other than $\pi^{0}$ will be analyzed, which will provide complementary results.

\section{References}

[1] Slides: http://indico. cern. ch/contributionDisplay . py? contribId=141\&sessionId=4\&conf Id=9499

[2] Research plan for spin physics at RHIC, BNL-73798-2005 (2005)

[3] K.Adcox et al.: Nucl. Inst. Meth. A499, 469 (2003)

[4] A. Adare et al.: Phys. Rev. Lett. 97, 252002 (2006); S. S. Adler et al.: Phys. Rev. Lett. 91, 241803 (2003); S. S. Adler et al.: Phys. Rev. Lett. 98, 012002 (2007)

[5] K. A. Dress and Z. Xu, "Proceedings of the PAC2001 Conference,",3120 (2001)

[6] B. Jäger et al.: Phys. Rev. D67, 054005 (2003); M. Glück et al.: Phys. Rev. D63, 094005 (2001).

[7] T.-c. Meng, J.-c. Pan, Q.-b. Xie, and W. Zhu: Phys. Rev. D40, 769 (1989). 